\title{
Modified Guidance Laws to Escape Microbursts with Turbulence
}

\author{
ATILLA DOGAN* and PIERRE T. KABAMBA ${ }^{\dagger}$ \\ Department of Aerospace Engineering, University of Michigan, 1320 Beal, \\ Ann Arbor, Ml 48109-2140
}

(Received 7 September 2001)

\begin{abstract}
This paper introduces Modified Altitude- and Dive-Guidance laws for escaping a microburst with turbulence. The goal is to develop a procedure to estimate the highest altitude at which an aircraft can fly through a microburst without running into stall. First, a new metric is constructed that quantifies the aircraft upward force capability in a microburst encounter. In the absence of turbulence, the metric is shown to be a decreasing function of altitude. This suggests that descending to a low altitude may improve safety in the sense that the aircraft will have more upward force capability to maintain its altitude. In the presence of stochastic turbulence, the metric is treated as a random variable and its probability distribution function is analytically approximated as a function of altitude. This approximation allows us to determine the highest safe altitude at which the aircraft may descend, hence avoiding to descend too low. This highest safe altitude is used as the commanded altitude in Modified Altitude- and Dive-Guidance. Monte Carlo simulations show that these Modified Altitude- and Dive-Guidance strategies can decrease the probability of minimum altitude being lower than a given value without significantly increasing the probability of crash.
\end{abstract}

Key words: Microburst; Windshear; Aircraft dynamics; Flight safety; Turbulence; Monte Carlo method; Probabilistic analysis

\section{INTRODUCTION AND PROBLEM STATEMENT}

Microbursts are a hazard for landing aircraft, and associated escape strategies have been studied in the literature (see Ref. [1] for a list of related references). Among these studies, only Refs. $[5,11,13,14]$ suggest that an initial descent may improve the safety of an escape maneuver. However, Refs. $[5,11,13]$ use point-mass aircraft models and do not consider the effect of turbulence, stall prevention action, or air vorticity on escape performance.

A recent study [14], using a 6-DOF aircraft model and taking into account the effects of turbulence, stall prevention and air vorticity, showed that the probability of crash decreases as the commanded altitude decreases as long as the commanded altitude is higher than an optimal value. However, for moderate microburst, it may be possible to safely recover with a higher commanded altitude. On the other hand, an attempt to fly too high, with AltitudeGuidance, causes the aircraft to stall and, with Dive-Guidance, results in an even lower minimum altitude. This, in return, increases the risk of crash. The goal of this work is to

\footnotetext{
*E-mail: adogan@engin.umich.edu

† E-mail: kabamba@engin.umich.edu
} 
improve upon [14]; specifically when a microburst is encountered, to determine the highest altitude that an aircraft can maintain without running into stall and to fly the aircraft at that altitude. This highest altitude, called "critical altitude", $h_{\mathrm{cr}}$ in this paper, will, of course, depend on the aircraft energy state and the strength of the microburst and the turbulence. Note that Ref. [3] provides a formula for selecting the target altitude, based on optimal trajectory studies of a point-mass model of an aircraft flying in a vertical plane through a microburst without turbulence.

Since the critical altitude, by definition, is the highest commanded altitude with which the aircraft with Altitude-Guidance can fly though a microburst without running into stall, it can be determined by using the full, non-linear, 6-DOF flight simulator developed in [14]. However, the goal here is to develop a procedure to estimate the critical altitude that can be used onboard to decide on the commanded altitude for Altitude- or Dive-Guidance. This paper provides a procedure to determine the critical altitude for a microburst encounter, depending on the strength of microburst and the aircraft energy state. Thus, the aircraft is prevented both from descending unnecessarily to an optimal altitude and from flying so high that it runs into stall. This goal is achieved by utilizing a new quasi-static lift-capability factor constructed in this paper.

Before presenting details, we show a comparison of the critical altitude determined by the flight simulator and its estimation by our procedure. Figures 1 and 2 show the comparison in different microbursts obtained by varying $f_{h}$. and $f_{r}$, respectively. The figures show that the estimation procedure captures the trend of the critical altitude, that is, as the microburst becomes stronger the critical altitude decreases. Both figures suggest that the procedure always underestimate the critical altitude. Although underestimation means the commanded altitude will be lower, i.e. the aircraft would fly through the microburst safely with commanded altitude higher than the estimate of the critical altitude, there is no risk of attempting to fly higher than the critical altitude, which would increase the probability of crash overall [14]. Underestimation is due to the fact that a "quasi-static" lift-capability factor is used for a

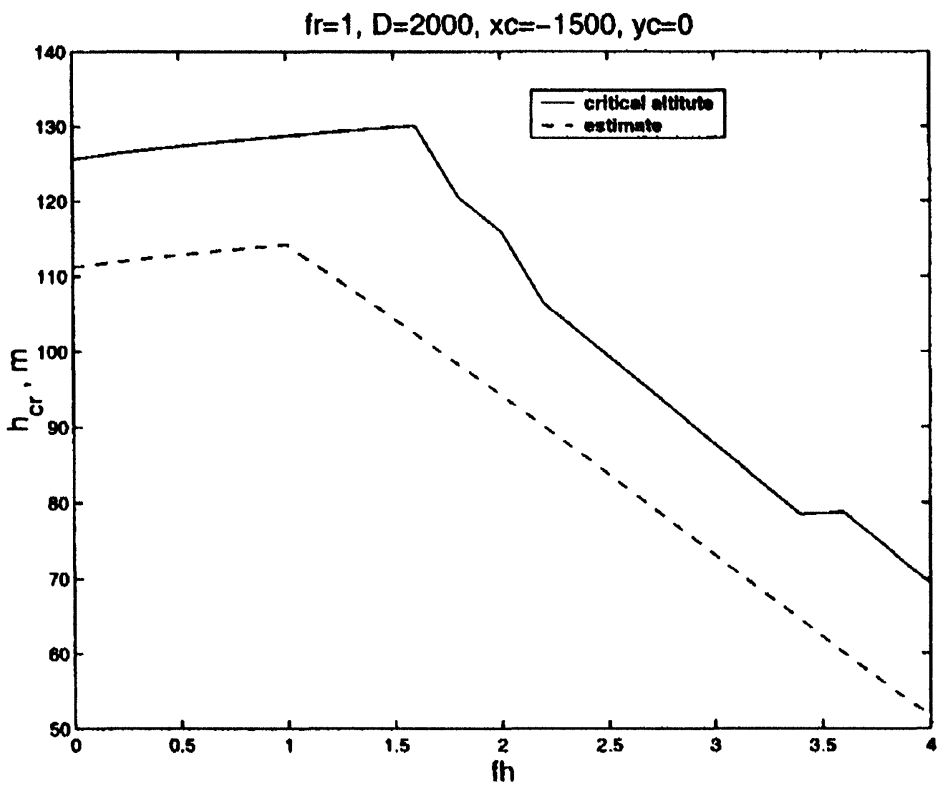

FIGURE 1 The critical altitude and its estimate $v s . f_{h}$. 


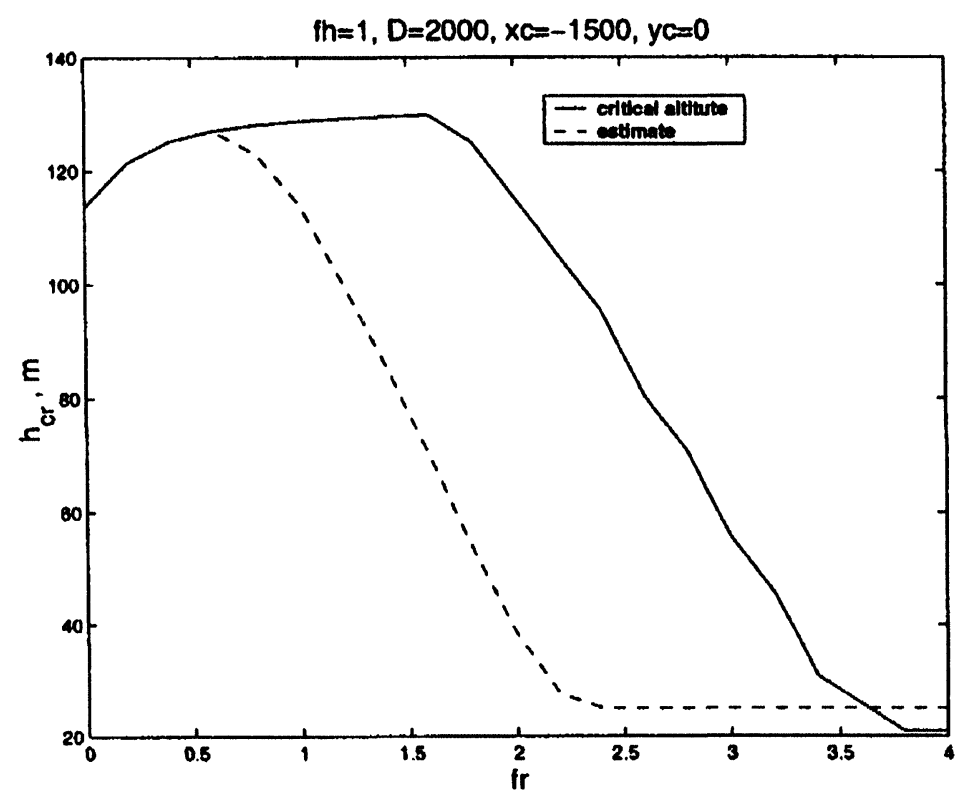

FIGURE 2 The critical altitude and its estimate $v s . f_{r}$.

very dynamic problem. The main reason to use a quasi-static factor is to find a procedure simple enough to be used onboard to estimate the critical altitude.

In this study, we first define this lift-capability factor, that quantifies the upward force capability of the aircraft, and show that, in the absence of turbulence, it is a decreasing function of altitude. Then, in the presence of stochastic turbulence, we treat the lift-capability factor as a random variable, and its probability density function (PDF) is approximated analytically as a function of altitude. This analytical approximation allows us to estimate the critical altitude in the sense that the aircraft flies with minimum, or prespecified, probability of failing to have enough vertical force capability to maintain the altitude. This critical altitude is used as commanded altitude in the Modified Altitude and Dive Guidance Strategies that we propose. Our results suggest that, under the assumptions of this paper, altitude- and dive-guidance can be improved by this modification, in that we can decrease the probability of minimum altitude being less than a given value without compromising the probability of crash. Hence, in a microburst encounter, the critical altitude can be computed using information on the microburst and aircraft energy state, and the aircraft can be guided to fly at that altitude with minimum or prespecified probability of failing to have enough vertical force capability. Like in Ref. [14], Modified Altitude-Guidance appears to perform better than the other escape strategies.

\section{MATHEMATICAL MODEL}

The equations of longitudinal aircraft motion (including the effect of windshear) used in this paper are equivalent to those given in Ref. [14]. Aircraft data and aerodynamic coefficients used in this study are representative of a large, jet-engine commercial transport airplane in landing configuration [15]. 
The equations of translational dynamics and kinematics are written in terms of $V$, airspeed, $\alpha$, angle-of-attack, $x$, longitudinal distance from the runway threshold and $z$, vertical downward direction into the ground:

$$
\begin{gathered}
\dot{V}=-g \sin (\theta-\alpha)+\frac{1}{m}[-D+T \cos (\alpha+\delta)] \\
-\dot{W}_{x} \cos (\theta-\alpha)+\dot{W}_{z} \sin (\theta-\alpha), \\
\dot{\alpha}=q+\frac{g}{V} \cos (\theta-\alpha)-\frac{1}{m V}[L+T \sin (\alpha+\delta)] \\
-\frac{\dot{W}_{x}}{V} \sin (\theta-\alpha)-\frac{\dot{W}_{z}}{V} \cos (\theta-\alpha), \\
\dot{x}=V \cos (\theta-\alpha)+W_{x}, \\
\dot{z}=-V \sin (\theta-\alpha)+W_{z},
\end{gathered}
$$

where $g$ is the acceleration of gravity, $\delta$ is the angle between thrust direction and zero-lift axis, and $m$ is the mass of the aircraft. The equations of rotational dynamics and kinematics, in terms of $q$, angular velocity around body $y$-axis, and $\theta$, pitch angle, are

$$
\begin{gathered}
\dot{q}=\frac{\mathcal{M}}{I_{y y}}, \\
\dot{\theta}=q,
\end{gathered}
$$

where $I_{y y}$ is the moment of inertia around body $y$-axis. The equation for engine dynamics, in terms of $\xi$, throttle response, is

$$
\dot{\xi}=\frac{1}{\tau}\left(\xi_{t}-\xi\right)
$$

where $\xi_{t}$ is the throttle setting. The above equations are based on the following assumptions: (i) the wind flow is steady, (ii) the earth is flat and non-rotating, (iii) the aircraft weight is constant, (iv) the flight is in a vertical plane. The thrust $T$ is assumed to have a fixed inclination $\delta$ relative to the zero-lift axis, but the thrust vector remains in the aircraft plane of symmetry.

The throttle response is modeled as a first-order lag with time constant $\tau$. The maximum thrust, $T_{\max }$, is assumed to be a function of airspeed only, i.e.

$$
T=\xi T_{\max }
$$

where

$$
T_{\max }=T_{0}+T_{1} V+T_{2} V^{2}
$$


The aerodynamic drag and lift forces and their coefficients are

$$
\begin{aligned}
& D=\frac{1}{2} \rho V^{2} \mathcal{S} C_{D}, \\
& L=\frac{1}{2} \rho V^{2} \mathcal{S} C_{L},
\end{aligned}
$$

where [15]

$$
\begin{aligned}
& C_{D}=C_{D_{0}}+C_{D_{\alpha}} \alpha, \\
& C_{L}=C_{L_{0}}+C_{L_{\alpha}} \alpha+C_{L_{q}} \frac{\bar{c}}{2 V} q+C_{L_{\delta_{e}}} \delta_{e},
\end{aligned}
$$

where $\rho$ is air density, $\mathcal{S}$ is wing area and $\delta_{e}$ is the elevator deflection. The aerodynamic pitching moment and its coefficient are

$$
\mathcal{M}=\frac{1}{2} \rho V^{2} \mathcal{S} \bar{c} C_{\mathcal{M}}+T \Delta z
$$

where [15]

$$
C_{\mathcal{M}}=C_{\mathcal{M}_{\alpha}} \alpha+C_{\mathcal{M}_{\delta_{e}}} \delta_{e}+C_{\mathcal{M}_{q}} \frac{\bar{c}}{2 V} q
$$

where $\bar{c}$ is the chord and $\Delta z$ is the moment arm of thrust around body $y$-axis. Note that the effect of thrust on pitching moment is taken into account. In the mathematical model the control variables are: thrust setting, $\xi_{t}$; and elevator deflection, $\delta_{e}$. They are constrained by

$$
\begin{gathered}
0<\xi_{t} \leq 1, \\
-20^{\circ} \leq \delta_{e} \leq 20^{\circ} .
\end{gathered}
$$

The 1-g stall speed of a large airliner jet with weight of 564,000 lbs and $30^{\circ}$ flap setting (gear down) is $61 \mathrm{~m} / \mathrm{s}$ [16]. If airspeed drops to the stall speed while angle-of-attack, $\alpha$, is greater than $\alpha_{\max }$, (which is $17.2^{\circ}[3]$ ), the aircraft is assumed to stall.

The microburst model [5] used herein is axisymmetric, three-dimensional, and stationary. The induced radial and vertical deterministic wind velocities at any point in three-dimensional space can be computed through the following relations

$$
\begin{gathered}
W_{r}^{d}=f_{r} \cdot\left(\frac{100}{[(r-D / 2) / 200]^{2}+10}-\frac{100}{[(r+D / 2) / 200200]^{2}+10}\right), \\
W_{h}^{d}=-f_{h} \cdot\left(\frac{0.4 h}{(r / 400)^{4}+10}\right),
\end{gathered}
$$

where

$$
r=\sqrt{\left(x-x_{c}\right)^{2}+y_{c}^{2}}
$$

where $h$ is altitude, $f_{r}$, is intensity of the horizontal shear, $f_{h}$ is intensity of the downdraft, $r$ is the radial distance from the microburst center, $D$ is the diameter of the peak radial 
outflow-velocity contour, and $\left(x_{c}, y_{c}\right)$ is the position of the microburst center in $(x, y)$ coordinates, and $W_{h}$ is positive in vertical upward direction. Using polar coordinates, the horizontal deterministic wind component, $W_{x}^{d}$, can be readily related to the radial wind component, $W_{r}^{d}$ :

$$
W_{x}^{d}=\cos \psi_{w} W_{r}^{d}(r)
$$

where $\psi_{w}$ is the angle between the direction of radial wind and $x$-axis. Stochastic turbulence is superimposed on the deterministic wind components of Eqs. (18) and (20). Thus,

$$
W_{h}=W_{h}^{d}+d_{h}, \quad W_{x}=W_{x}^{d}+d_{x},
$$

where $d_{h}$ and $d_{x}$ are the turbulence components in vertical and horizontal directions, respectively. The turbulence components in inertial frame are related to those in body frame by a rotation matrix as follows:

$$
\begin{aligned}
& d_{x}=\cos \theta u_{g}+\sin \theta w_{g}, \\
& d_{h}=\sin \theta u_{g}-\cos \theta w_{g},
\end{aligned}
$$

where $u_{g}$ and $w_{g}$ are turbulence components in body $x$-and $z$-axis, respectively. Turbulence is modeled using the Dryden Power Spectral Density (PSD) functions. State variable models for $u_{g}$ and $w_{g}$, respectively, are [17]

$$
\begin{gathered}
\dot{u}_{g}=-\lambda_{u} u_{g}+\sqrt{K_{u}} \eta_{1}, \\
{\left[\begin{array}{c}
\dot{x}_{1} \\
\dot{x}_{2}
\end{array}\right]=\left[\begin{array}{cc}
0 & 1 \\
-\lambda_{w}^{2} & -2 \lambda_{w}
\end{array}\right]\left[\begin{array}{l}
x_{1} \\
x_{2}
\end{array}\right]+\left[\begin{array}{l}
0 \\
1
\end{array}\right] \eta_{2},} \\
w_{g}=\sqrt{K_{w}}\left[\beta_{w}, 1\right]\left[\begin{array}{l}
x_{1} \\
x_{2}
\end{array}\right],
\end{gathered}
$$

where $\eta_{1}$ and $\eta_{2}$ are signals from independent white noise sources. The intensity of the $w$-component of turbulence in body-fixed frame $\left(\sigma_{w_{g}}\right)$ is chosen to be 4 , which corresponds to severe storm. The intensities of the other components are computed using the assumption that the intensities of the three translational components of turbulence are isotropic. All the coefficients in Eqs. (24), (25) depend on altitude through so-called turbulence scale lengths [17]:

$$
L_{w_{g}}=h, \quad L_{u_{g}}=145 h^{1 / 3} .
$$

In this microburst model with turbulence, we assume that turbulence and microburst windshear can be modeled independently. Experimental evidence suggests that turbulence length scales increase through a microburst and, in some unknown manner, depend on microburst size and strength [18]. Such a functional dependence between turbulence and microburst parameters will result in a nonstationary and non-Gaussian wind process description [10]. This functional dependence is neglected in this study. 


\section{DETERMINISTIC ANALYSIS}

\subsection{Construction of a Lift-capability Metric}

Upon encountering a microburst, in the absence of turbulence, descending to a lower altitude generally results in safer recovery because of two separate reasons: (i) the special structure of the wind field in a microburst and (ii) trading altitude for airspeed.

The first mechanism can be understood as follows. In a microburst, the air moves downward, causing a downdraft and, near the ground, spreads outward, resulting in horizontal windshear. The velocity of downdraft decreases with altitude due to the stagnation level on the ground. Thus, flying at a lower altitude through the microburst will expose the aircraft to downdraft of smaller magnitude.

As the second mechanism, once the escape maneuver is initiated, altitude is traded for airspeed and the aircraft flies with high airspeed through the core of the microburst, where the downdraft is most severe. The benefit of this mechanism can be understood by considering the effect of the microburst on aerodynamic lift, which can be reduced by windshear in two ways. First, a wind speed change along the flight path will produce a direct initial effect on lift in proportion to the change in airspeed. Second, a vertical windshear will change lift by changing the angle of attack directly without affecting airspeed initially. A windshear that acts to reduce airspeed or angle of attack will reduce aerodynamic lift and result in a deteriorating flight path [2]. Thus, if an aircraft enters a microburst with higher airspeed gained by trading off altitude, the airspeed fluctuation due to windshear will be smaller relative to the original airspeed. Additionally, angle of attack changes due to the vertical windshear will be smaller than when flying with low airspeed. Therefore, aerodynamic lift reduction due to the windshear and downdraft will be relatively small in the case of higher airspeed.

Now, we will define a lift-capability metric, $L_{f}$, that quantifies the aircraft maximum capability of generating an upward force, and compare different escape trajectories using this metric. The lift-capability metric presumes the following quasi-static scenario in the absence of turbulence: The aircraft is flying horizontally in the microburst, i.e. the instantaneous absolute velocity of the aircraft is horizontal, and the flight is longitudinal. Under these conditions we compute the maximum force that would be available to initiate a climb.

Note that $W_{h}=W_{h}^{d}$ and $W_{x}=W_{x}^{d}$ due to the assumption, in this section, that there is no turbulence. As can be seen from Figure 3, the sum of the vertical forces, except the weight of the aircraft, with the positive direction being upward, is

$$
F_{v}=L \cos (\theta-\alpha)-D \sin (\theta-\alpha)+T \sin \theta .
$$

Since maximum thrust is used in microburst escape maneuvers, the only way to change the vertical force is to change the pitch angle, $\theta$. So, we define the lift-capability metric as the ratio of the maximum available $F_{v}$ to the weight, $W$ :

$$
L_{f}=\frac{\max _{\theta}\left(F_{v}\right)}{W} .
$$

To evaluate $\max _{\theta}\left(F_{v}\right)$ we need to write $F_{v}$ as a function of $\theta$. Using the assumptions that the flight is longitudinal and the inertial velocity of the aircraft, $V_{A / C}$ is horizontal, i.e. $\dot{x}=V_{A / C}$ and $\dot{z}=0$, Eqs. (3) and (4) yield

$$
\begin{aligned}
& \cos (\theta-\alpha)=\frac{V_{A / C}-W_{x}}{V} \\
& \sin (\theta-\alpha)=\frac{W_{h}}{V} .
\end{aligned}
$$




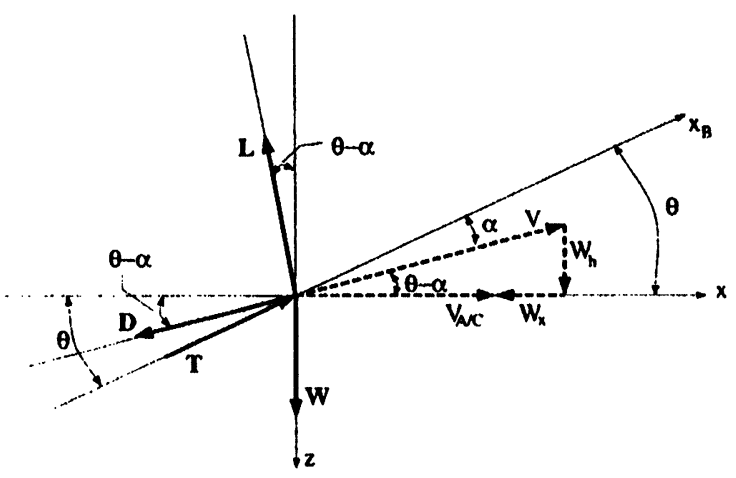

FIGURE 3 Velocities and forces in inertially horizontal longitudinal flight.

Substituting $L$ from (11) and (13), $D$ from (10) and (12) into Eq. (27) and recalling that the maximum thrust is used, Eq. (27) becomes

$$
\begin{aligned}
F_{v}= & \frac{1}{2} \rho V^{2} \mathcal{S}\left\{\left[C_{L_{0}}+C_{L_{\alpha}} \alpha+C_{L \delta_{e}} \delta_{e}\right]\left(\frac{V_{A / C}-W_{x}}{V}\right)\right. \\
& \left.-\left[C_{D_{0}}+C_{D_{\alpha}} \alpha\right] \frac{W_{h}}{V}\right\}+\left(T_{0}+T_{1} V+T_{2} V^{2}\right) \sin \theta .
\end{aligned}
$$

Note that since the analysis here is quasi-static, the velocity terms, e.g. in $C_{L}$ in Eq. (13) is neglected. The assumptions of quasi-static analysis and longitudinal flight, together with Eq. (5) imply that

$$
\mathcal{M}=0
$$

Hence

$$
\delta_{e}=-\frac{1}{C_{\mathcal{M} \delta_{e}}}\left[C_{\mathcal{M} \alpha} \alpha+\frac{2\left(T_{0}+T_{1} V+T_{2} V^{2}\right) \Delta z}{\rho \mathcal{S} \bar{c} V^{2}}\right]
$$

From Eq. (30), the angle-of-attack for a given pitch angle, in the case of horizontal flight, is

$$
\alpha=\theta-\arcsin \left(\frac{W_{h}}{V}\right)
$$

Thus, Eq. (31) along with Eqs. (33) and (34) give $F_{v}$ as a function of $\theta$. Now, the problem of maximizing $F_{v}(\theta)$ can be solved. It can numerically be shown that both $F_{v}(\theta)$ and $\alpha(\theta)$ are increasing functions of $\theta$. Thus, recalling that the angle-of-attack has a maximum bound, we obtain

$$
\max _{\theta}\left(F_{v}\right)=F_{v}\left(\theta^{*}\right)
$$

such that

$$
\alpha\left(\theta^{*}\right)=\alpha_{\max }
$$


Therefore, the the lift-capability factor, $L_{f}$ is

$$
\begin{aligned}
L_{f}= & \frac{\rho V^{2} \mathcal{S}}{2 W}\left[\left(C_{L_{0}}+C_{L_{\alpha}} \alpha_{\max }+C_{L \delta_{e}} \delta_{e}^{*}\right) \frac{V_{A / C}-W_{x}}{V}\right. \\
& \left.-\left(C_{D_{0}}+C_{D_{\alpha}} \alpha_{\max }\right) \frac{W_{h}}{V}\right]+\frac{\left(T_{0}+T_{1} V+T_{2} V^{2}\right)}{W} \sin \theta^{*},
\end{aligned}
$$

where

$$
\begin{aligned}
\theta^{*} & =\alpha_{\max }+\arcsin \left(\frac{W_{h}}{V}\right), \\
\delta_{e}^{*} & =-\frac{C_{\mathcal{M} \alpha} \alpha_{\max }+\left(2\left(T_{0}+T_{1} V+T_{2} V^{2}\right) \Delta z\right) / \rho \mathcal{S} \bar{c} V^{2}}{C_{\mathcal{M} \delta_{e}}},
\end{aligned}
$$

where $\theta^{*}$ is the pitch angle that maximizes $F_{v}$ and $\delta_{e}^{*}$ is the elevator deflection when $F_{v}$ is maximum. The lift-capability factor $L_{f}$ is constructed to determine whether an aircraft, initially flying horizontally, has any climb capability. Roughly speaking if $L_{f}>1$, then the aircraft has enough vertical force capability to climb, but if $L_{f}<1$, the aircraft does not have enough vertical force to maintain its altitude.

\subsection{Lift-capability of Low Altitude Flight}

Now, for a given specific inertial energy, we will write $L_{f}$ as a function of altitude and analyze how it changes with altitude. The specific inertial energy is defined as

$$
E_{i}=\frac{1}{2} V_{A / C}^{2}+g h
$$

For a given inertial energy, we can write the inertial aircraft speed as a function of altitude:

$$
V_{A / C}=\sqrt{2\left(E_{i}-g h\right)}
$$

Since $\vec{V}_{A / C}=\vec{W}_{w}+\vec{V}$, from Figure 3, we obtain

$$
V=\sqrt{\left(V_{A / C}-W_{x}\right)^{2}+W_{h}^{2}}
$$

Thus, airspeed $V$ is also written as a function of position since $W_{h}$ is a function of $x$ and $h$, and $W_{x}$, is a function of $x$. Hence, substituting $V_{A / C}(h), V(x, h), W_{x}(x)$, and $W_{h}(x, h)$ into Eqs. (37), (38), and (39), $L_{f}$ becomes a function of position, $(x, h)$.

In Figures 4 and $5, L_{f}$ is plotted as a function of altitude at various horizontal positions, for the headwind and tailwind cases, respectively. Both figures show that $L_{f}$ is a decreasing function of altitude for the particular airplane and microburst considered. A formal proof of the generality of this statement is in Ref. [1]. Figure 4 shows that for a given altitude, headwind is performance-increasing. The upper curve corresponds to the position with maximum headwind. The lower curve corresponds to the center of the microburst, where there is theoretically no horizontal wind. On the other hand, Figure 5 shows that for a given altitude, the tailwind region is where the aircraft is in real danger and the most dangerous position is where the tailwind magnitude reaches its peak. 


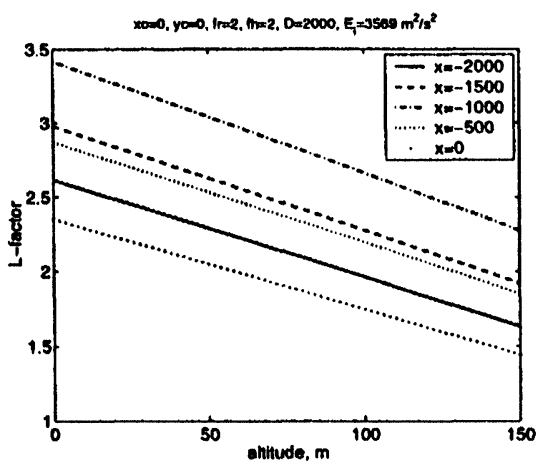

FIGURE $4 L_{f}$ as a function of altitude, plotted for various horizontal positions, in the case of headwind.

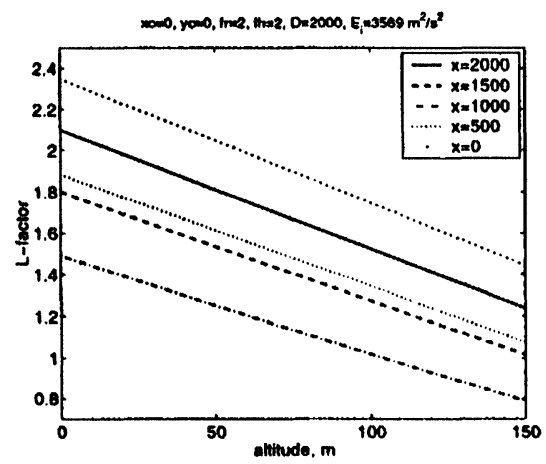

FIGURE $5 L_{f}$ as a function of altitude, plotted for various horizontal positions, in the case of tailwind.

\section{PROBABILISTIC ANALYSIS}

\subsection{Construction of a Probabilistic Lift-capability Factor}

In the previous section, we have shown that in the absence of turbulence, the lift-capability factor is a decreasing function of altitude. This implies that the lower the aircraft flies the safer the escape maneuver will be in terms of vertical force capability. In this section, we will investigate the lift capability of low altitude flight in the presence of turbulence through the lift-capability factor, $L_{f}$. In the previous section, $L_{f}$ was a deterministic function since turbulence was not taken into account. However, in this section, we will reconstruct $L_{f}$ as a random variable to study the effect of turbulence on the lift-capability of low altitude flight.

Equations (37)-(39) imply that $L_{f}$ is a function of the wind components, $W_{x}$ and $W_{h}$. Also note from Eq. (21) that, in the presence of turbulence, the wind components are random processes due to the turbulence components, given in Eqs. (24) and (25). However, recall that $L_{f}$ has been constructed under the assumption of a quasi-static analysis. Consistent with this assumption, we replace the stationary random processes $u_{g}(t), w_{g}(t)$ of Eqs. (24), (25) by random variables $u_{g}, w_{g}$ with same mean and variance. Specifically we have (see Appendix A for detail)

$$
E\left[u_{g}\right]=0,
$$




$$
\begin{gathered}
E\left[w_{g}\right]=0, \\
\operatorname{var}\left(u_{g}\right)=\frac{145 \sigma^{2} \sigma_{w_{g}}^{2}}{\pi} \frac{1}{h^{2 / 3}}, \\
\operatorname{var}\left(w_{g}\right)=\frac{\sigma^{2}}{\pi} \sigma_{w^{2}}^{2} .
\end{gathered}
$$

Additionally, Ref. [14] showed that the effect of high frequency components of wind is not seen on the pitch angle of the aircraft. Thus, unlike Eq. (38), we assume, for simplicity, that the pitch angle is only function of deterministic components of wind, i.e.

$$
\theta^{*}=\alpha_{\max }+\arcsin \left(\frac{W_{h}^{d}}{V^{d}}\right)
$$

where, from Eq. (42)

$$
V^{d}=\sqrt{\left(V_{A / C}-W_{x}^{d}\right)^{2}+\left(W_{h}^{d}\right)^{2}}
$$

Hence we have constructed the lift-capability factor as a random variable: $L_{f}$ is given in Eq. (37) as a function of $V, \delta_{e}^{*}, V_{A / C}, W_{x}, W_{h}$, and $\theta^{*} . V$ is given in Eq. (42); $\delta_{e}^{*}$ in Eq. (39); $V_{A / C}$ in (41); $W_{x}$ and $W_{h}$ in (21); and $\theta^{*}$ in (47).

\subsection{Statistical Properties of the Lift-capability Factor}

The lift-capability factor $L_{f}$ is now treated as a random variable because it is a function of two Gaussian random variables, $u_{g}$ and $w_{g}$. Specifically, for given microburst parameters, inertial specific energy, and spatial position, we have

$$
L_{f}=L_{f}\left(u_{g}, w_{g}\right) .
$$

Thus, a logical next step is to find the probability density function (PDF) of $L_{f}$ in terms of the PDF's of $u_{g}$ and $w_{g}$. Unfortunately, the function, $L_{f}\left(u_{g}, w_{g}\right)$, is nonlinear and it would be difficult to analytically determine its exact PDF. Instead, we will find an analytical approximation as follows.

The Taylor series expansion of $L_{f}\left(u_{g}, w_{g}\right)$ is

$$
L_{f}\left(u_{g}, w_{g}\right)=L_{f}(0,0)+\left.\frac{\partial L_{f}}{\partial u_{g}}\right|_{(0,0)} u_{g}+\left.\frac{\partial L_{f}}{\partial w_{g}}\right|_{(0,0)} w_{g}+\text { H.O.T. }
$$

Since the turbulent components of wind are high-frequency fluctuations and small relative to the deterministic components, $u_{g}$ and $w_{g}$ are assumed to be small. Hence, in Eq. (50), the higher order terms are neglected. The partial derivatives $\partial L_{f} /\left.\partial u_{g}\right|_{(0,0)}$ and $\partial L_{f} /\left.\partial w_{g}\right|_{(0,0)}$ are given in Appendix B. With this linear approximation, $L_{f}$ is also a Gaussian random variable with [20]

$$
\begin{aligned}
E\left[L_{f}\right] & =L_{f}(0,0), \\
\operatorname{var}\left(L_{f}\right) & =\left.\frac{\partial L_{f}}{\partial u_{g}}\right|_{(0,0)} ^{2} \operatorname{var}\left(u_{g}\right)+\left.\frac{\partial L_{f}}{\partial w_{g}}\right|_{(0,0)} ^{2} \operatorname{var}\left(w_{g}\right)
\end{aligned}
$$


where $L_{f}(0,0)$ is the lift-capability factor in the absence of turbulence in Eq. (37) and was shown to be a function of altitude for given specific inertial energy and horizontal position. It can be shown that $\operatorname{var}\left(L_{f}\right)$ is also a function of altitude for given specific inertial energy and horizontal position.

To see how accurate the linear approximation of $L_{f}$ is, we compare the mean (51) and variance (52) of the linear approximation with the minimum variance unbiased estimates [20] of the mean and variance of $L_{f}$ in Eq. (49) under the assumption that $L_{f}$ is a Gaussian random variable. Figure 6 shows that the linear approximation almost exactly matches the estimates for the whole altitude range of interest. For different microburst cases a similar consistency was obtained but not shown here. Therefore, in the remainder of the paper, we will use the linear approximation instead of the fully nonlinear $L_{f}$.

Since $L_{f}$ can be approximated as a Gaussian random variable with known mean (51) and variance (52), its PDF is known. Thus, the probability of $L_{f}$ being less than or equal to a given value, $p_{L_{f}}$, is

$$
P\left[L_{f} \leq p_{L_{f}}\right]=\frac{1}{2}\left[1+\operatorname{erf}\left(\frac{p_{L_{f}}-E\left[L_{f}\right]}{\sqrt{2 \operatorname{var}\left(L_{f}\right)}}\right)\right],
$$

where erf(.) is the "error function" [20],

$$
\operatorname{erf}(x)=\frac{2}{\sqrt{\pi}} \int_{0}^{x} \mathrm{e}^{-t^{2}} \mathrm{~d} t
$$

Recall that $E\left[L_{f}\right]$ and $\operatorname{var}\left(L_{f}\right)$ are functions of altitude. Thus, $P\left[L_{f} \leq p_{L_{f}}\right]$ is also a function of altitude for given specific inertial energy and horizontal position in a microburst.

Figure 7 shows $P\left[L_{f} \leq p_{L_{f}}\right]$ as a function of altitude for various horizontal positions in a microburst. The figure suggests that the most dangerous horizontal position in a microburst is where the mean of the tailwind reaches its peak. This is because the probability of $L_{f}$ being less than 1 is higher at this position than at any other position for any altitude. Recall that in Subsection 3.2, the deterministic analysis of $L_{f}$ in the absence of turbulence yielded the same
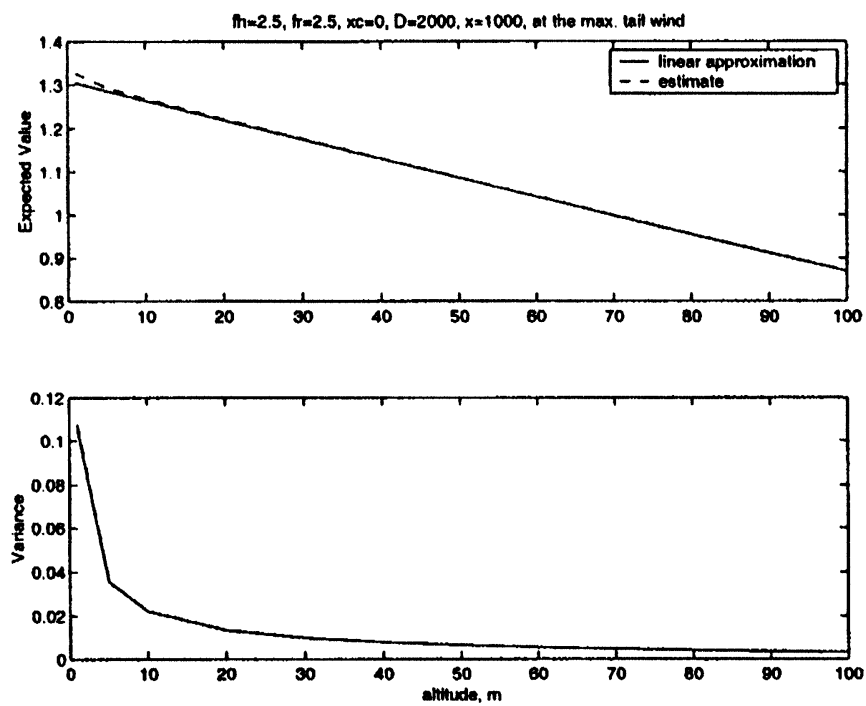

FIGURE 6 Mean and variance of the linear approximation and estimate of $L_{f}$ in a microburst for various altitudes at the horizontal location where tail wind is maximum. 


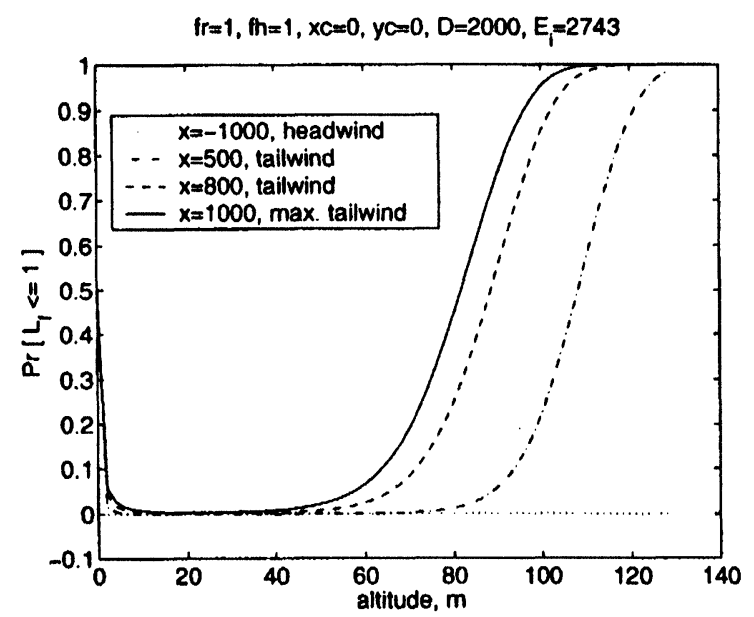

FIGURE 7 Probability of $L_{f}$ being less than $1 \mathrm{vs}$. altitude for various horizontal positions.

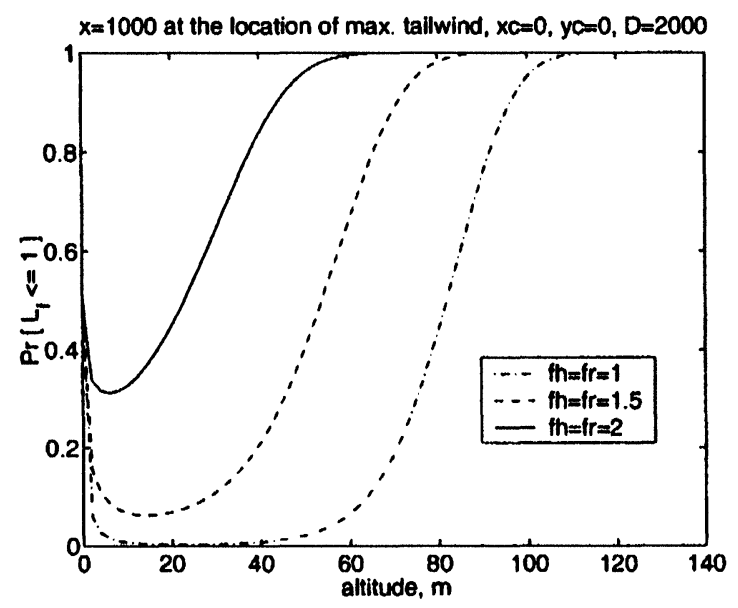

FIGURE 8 Probability of $L_{f}$ being less than $1 \mathrm{vs}$. altitude for various microburst cases.

conclusion. Thus, in the remainder of the paper, by $P\left[L_{f} \leq p_{L_{f}}\right]$ we will mean the probability of $L_{f}$ being less than $p_{L_{f}}$ at the horizontal location where the tailwind is maximum, unless otherwise stated.

Figure 8 shows the effect of microburst strength parameters on the probability of $L_{f}$ being less than 1. As the microburst becomes stronger, the flight at any altitude becomes less safe in the sense that the probability of $L_{f}$ being less than 1 increases.

Both Figures 7 and 8 show the superiority of the statistical analysis over the deterministic analysis. While the deterministic analysis showed the advantage of descending in a microburst encounter, it completely failed to show that descending too low may increase the risk of failing to have enough vertical force capability to maintain the altitude. However, Figures 7 and 8 clearly show that flying lower increases lift capability up to an optimal 
altitude, and that flying lower than this optimal value dramatically decreases the lift capability. This is because $L_{f}$ being less than 1 means that the aircraft does not have enough vertical force capability to maintain its altitude. Thus, we have analytically corroborated the results of the Monte Carlo simulation in [14]: the probability of crash decreases as the commanded altitude decreases as long as the commanded altitude is higher than an optimal value.

\section{CRITICAL ALTITUDE IN A MICROBURST ESCAPE}

In a microburst escape maneuver, it has been shown that descending may improve safety. We have, in our previous work [14], shown that, in any microburst encounter, the probability of crash is minimized if the aircraft descends to an optimal altitude. However, in the case of moderate microbursts, it is possible to safely recover with commanded altitudes that are higher than the optimal commanded altitude. On the other hand, flying too high has been shown [14] to be dangerous as well. Hence, it is important to determine the critical altitude, which depends on the microburst strength and aircraft energy capacity. Thus, the goal of this section is to develop an algorithm that, using available information on the microburst and aircraft state variables, gives the critical altitude in a microburst encounter.

\subsection{Determining a Critical Altitude}

The lift-capability factor, developed in the previous sections, can show whether the aircraft has enough vertical force capacity to maintain flight at a given altitude. In the probabilistic analysis, it also took into account the effect of turbulence on the escape performance. In the deterministic approach, the lift-capability factor can be written explicitly as a function of altitude. In the probabilistic approach, its probability of being less than a given value can also be formulated as a function of altitude. Having these facts in mind, in the section, we develop techniques to determine how high an aircraft can fly in a microburst encounter.

Figure 9 shows the general profile of $P\left[L_{f} \leq p_{L_{f}}\right]$ as a function of altitude. As seen in the figure, $h^{*}$ denotes the altitude that minimizes the probability that $L_{f}$ is less than $p_{L_{f}}$, and $\bar{h}$ denotes the maximum altitude of which $P\left[L_{f} \leq p_{L_{f}}\right]$ is less than the sum of the minimum probability of $L_{f}$ being less than $p_{L_{f}}$ and an increment, $\Delta_{p}$. Using $h^{*}$ as the recovery altitude

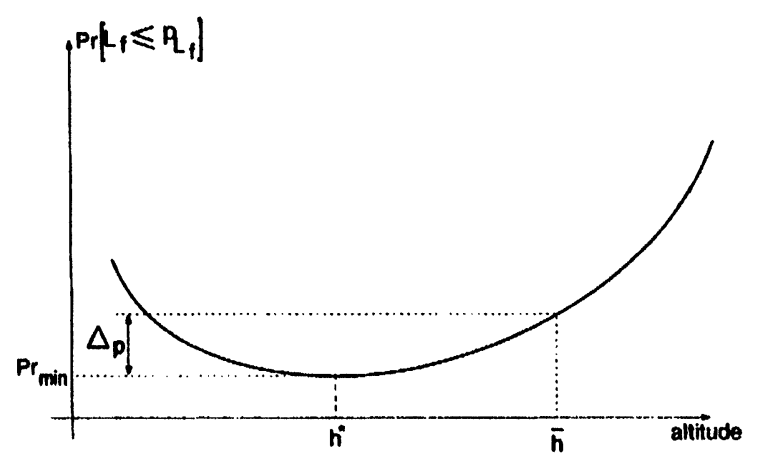

FIGURE 9 Graphical definition of $h^{*}$ and $\bar{h}$. 
will guarantee that the aircraft, in a microburst escape maneuver, flies with the minimum probability of failing to have enough vertical force capability to maintain its altitude, with a safety margin depending on the value of $p_{L_{f}}$. However, note that there will always be a positive probability of failing to have enough capability to maintain the altitude, unless $\operatorname{Pr}_{\text {min }}$ is zero. Flying at $\bar{h}$ means that the probability of $L_{f}$ being less than $p_{L_{f}}$ will always be smaller than $\operatorname{Pr}_{\min }+\Delta_{p}$.

The probability $P\left[L_{f} \leq p_{L_{f}}\right]$ is given in Eq. (53), and its dependence on altitude can be made explicit through Eqs. (37), (51), (52). These equations are then solved for $h^{*}$ and $\bar{h}$. Details are given in Appendix C.

\subsection{Microburst Escape Guidance Using the Lift-capability Factor}

Ref. [14] compares three different escape strategies, pitch-guidance, altitude-guidance and dive-guidance, in the following scenario. As the aircraft, guided by the landing controller, is descending, the F-factor [14] is monitored using data obtained from a reactive or forward-looking detection system. Once the F-factor exceeds the threshold value [14], the landing is aborted and an escape maneuver is immediately initiated. The sensitivity of the performances of the escape strategies with respect to variations in microburst size, strength and location, in the presence of turbulence, is analyzed using the Monte Carlo Method. The only data is assumed to be the statistical distribution of the microburst parameters and the comparison between different strategies is based on scalar performance measures: the probability of descending lower than a given altitude, and the probability of crash. The probability of descending lower than a given altitude is obtained from the PDF (Probability Distribution Function) of the minimum altitude, $h_{\min }$, reached in a microburst encounter. The probability of crash is the probability that the aircraft either runs into stall or has a ground contact during an escape maneuver. In the Monte Carlo Simulation, stall and ground contact are deemed equally dangerous and we assume that stall results in ground contact. Thus, the probability of crash is the PDF of minimum altitude evaluated at zero altitude.

The escape strategies analyzed in Ref. [14] are:

(i) Pitch-guidance: a $15^{\circ}$ target pitch angle is commanded and maintained throughout the microburst. This strategy is recommended in the Windshear Training Aid [2] by the FAA.

(ii) Dive-guidance: a $0^{\circ}$ target pitch angle is commanded until the altitude drops to the commanded altitude, $h_{c}$. Once the altitude becomes lower than $h_{c}$, a $15^{\circ}$ pitch angle is commanded for the remainder of the escape maneuver.

(iii) Altitude-guidance ( $h$-guidance): This guidance strategy flies the aircraft at a constant recovery altitude in the high shear region of the microburst. It has two consecutive modes. In the altitude-guidance mode, it directs the aircraft to a given commanded altitude and keeps it there. Then, in the climbing mode, it commands a $15^{\circ}$ pitch angle to move the aircraft up from its recovery altitude.

The Monte Carlo simulation in Ref. [14] shows that, with respect to the probability of crash, altitude- and dive-guidance may be better than pitch-guidance, and that the probability of crash is minimum if the commanded altitude is chosen at an optimal value in both altitudeand dive-guidance. However, as stated before, the aircraft may recover with higher commanded altitude than the optimal value in a less severe microburst. 
If the altitude $h^{*}$ or $\bar{h}$ is chosen as the commanded altitude in altitude- or dive-guidance, the commanded altitude will increase as the strength of the microburst decreases, as seen in Figure 8. Thus, we define the following modified altitude- and dive-guidance strategies:

(iv) Dive-guidance with $h^{*}$ (Lf-guidance-dive-star): Dive-guidance with commanded altitude computed using $h^{*}$ for each microburst encountered.

(v) Altitude-guidance with $h^{*}$ (Lf-guidance-star): Altitude-guidance whose commanded altitude is computed using $h^{*}$ for each microburst encountered.

In (iv) and (v), the commanded altitude is

$$
h_{c}= \begin{cases}h^{*}, & \text { when } h^{*}>25 \\ 25, & \text { otherwise. }\end{cases}
$$

The safety margin, $p_{L_{f}}$, in $h^{*}$ computation is 1 . Since Ref. [14] suggests that descending lower than $25 \mathrm{~m}$ increases the probability of crash, even when we obtain $h^{*}$ less than $25 \mathrm{~m}$, the aircraft is not directed lower than $25 \mathrm{~m}$.

(vi) Dive-guidance with $\bar{h}$ (Lf-guidance-dive-bar): Dive-guidance with commanded altitude computed using $\bar{h}$ for each microburst encountered.

(vii) Altitude-guidance with $\bar{h}$ (Lf-guidance-bar): Altitude-guidance with commanded altitude computed using $\bar{h}$ for each microburst encountered.

In (vi) and (vii), the commanded altitude is

$$
h_{c}= \begin{cases}\bar{h}, & \text { when } \bar{h}>25 \\ 25, & \text { otherwise. }\end{cases}
$$

The lower limit on the commanded altitude is imposed for the same reason as for $h^{*}$ in (55). In $\bar{h}$ computation, $\Delta_{p}$ is chosen as 0.01 and $p_{L_{f}}$ is

$$
p_{L_{f}}=\left\{\begin{array}{cl}
1.1+\frac{\Delta_{f}}{10}, & \text { when } \Delta_{f}>0 \\
1.1, & \text { otherwise. }
\end{array}\right.
$$

where

$$
\Delta_{f}=f_{h}-f_{r} .
$$

Simulation experiments has shown that increasing $p_{L_{f}}$, the safety margin, when $f_{h}$ is greater than $f_{r}$, improves the escape performance in the sense that the probability of having minimum altitude less than a given value is decreased without increasing the probability of crash.

The controllers [14], used for the strategies (i)-(iii), are also used for the modified strategies (iv)-(vii). As in Ref. [14], the performances of the escape strategies are compared using Monte Carlo simulations that produce estimates of the PDF of the minimum altitude and the probability of crash in a microburst whose statistical properties are known. The problem formulation for the Monte Carlo simulation and the statistical properties of the microburst parameters can be found in Ref. [14]. Although the Eqs. (1)-(7), for the longitudinal aircraft motion are used in the derivation of $L_{f}$, a full 6-DOF aircraft model, given in Ref. [14], is used in the Monte Carlo simulation so as to make a fair comparison between the strategies (i)-(iii), with a constant $h_{c}$ for every microburst encountered, and their modified versions, (iv)-(vii), which utilize the lift-capability factor to determine the commanded altitude for each microburst encountered.

The scenario for the modified escape strategies is as follows: As the aircraft is approaching on the glide path, the wind velocity components are measured using reactive and/or forwardlooking sensors. With these measurements and the microburst model with unknown 
parameters, the microburst parameters are computed. Then, using the microburst model with the estimated parameters, the deterministic wind components at the position where the tail wind would reach its maximum are computed. Simultaneously, altitude and aircraft inertial speed $\left(\sqrt{\dot{x}^{2}+\dot{y}^{2}+\dot{z}^{2}}\right)$ are measured and the specific inertial energy of the aircraft is computed. Using the measurement of wind, a sample of vertical wind component is obtained. With this sample, the variance is estimated. Then, using the methods developed in the previous subsection, $h^{*}$ or $\bar{h}$ is computed. The above estimation should be continuously performed on-line and $h^{*}$ or $\bar{h}$ should be updated as the aircraft is approaching on the glide path. When the F-factor exceeds the threshold, the last update of $h^{*}$ or $\bar{h}$ is used to decide the commanded altitude in altitude- or dive-guidance.

In any practical application of this procedure, the underlying assumptions should be borne in mind:

- The microburst encountered has a wind distribution similar to that of the microburst model used here.

- The accompanying turbulence has statistical properties similar to those of the turbulence model used.

- The aircraft model and data are accurate enough.

- The wind velocity components are measured on-board.

- The variance of the vertical component of turbulence is estimated on-board.

- Aircraft altitude and inertial speed are measured.

\subsection{Monte Carlo Simulation Results}

As stated above, the goal of the modified escape strategies is to avoid descending lower than necessary, but to fly at the highest safe altitude in a microburst encounter. Comparison of the PDFs of $h_{\min }$ for various escape strategies can reveal whether the goal has been achieved. Of course such comparisons are conclusive only when the associated confidence intervals are disjoint. We have performed such conclusive comparisons, but for the sake of clarity, we will only show confidence intervals at altitudes that are multiples of $20 \mathrm{~m}$.

Figure 10 shows the PDF of $h_{\min }$ of pitch-guidance, $h$-guidance with $25 \mathrm{~m}$ commanded altitude, and the modified altitude guidance strategies, together with $95 \%$ confidence. Pitch guidance is used as the baseline strategy since it is recommended by the FAA [2]. Since Ref. [14] shows that altitude-guidance with $25 \mathrm{~m}$ commanded altitude yields the minimum probability of crash, the modified strategies are compared with altitude-guidance with $h_{c}$ of $25 \mathrm{~m}$. Regarding the probability of crash, both Lf-guidance-star and Lf-guidance-bar are as good as $h$-guidance with $h_{c}$ of $25 \mathrm{~m}$ since the PDFs at zero altitude are almost the same. It is also obvious that all the three are much better than pitch-guidance. Regarding the probability of minimum altitude being less than a given value, among the three, Lf-guidance-bar is the best because its PDF is either about the same as, or smaller than, those of the other two for any given altitude. For example, at $h=40 \mathrm{~m}$, the PDF of $h_{\min }$ of $h$-guidance is 1 , that of Lf-guidance-star is 0.8 , and that of Lf-guidance-bar is about 0.55 . In other words, with Lf-guidance-bar, it is much less likely to have minimum altitude less than $40 \mathrm{~m}$. Moreover, the probability of crash of Lf-guidance-bar is about the same as those of $h$-guidance and Lf-guidance-star.

Another interpretation of the PDFs is as follows: The aircraft with $h$-guidance has descended lower than $40 \mathrm{~m}$ in all cases; with Lf-guidance-star, 80 times out of 100 microburst encounter; and with Lf-guidance-bar, just 55 times. Also note that, with all the three, the aircraft has crashed or run into stall about 15 times. Thus, we can conclude that, with $h$-guidance, the aircraft has unnecessarily descended lower than $40 \mathrm{~m} 45$ times 


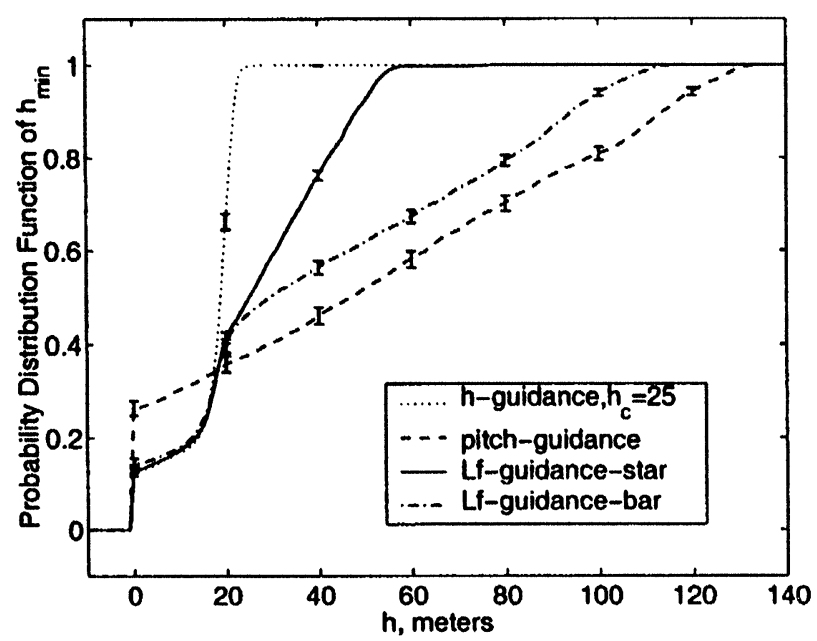

FIGURE 10 Probability distribution function (PDF) of $h_{\min }$, i.e. $\operatorname{Pr}\left(h_{\min } \leq h\right)$ of pitch-, altitude- and modifiedaltitude-guidance, with $95 \%$ confidence intervals.

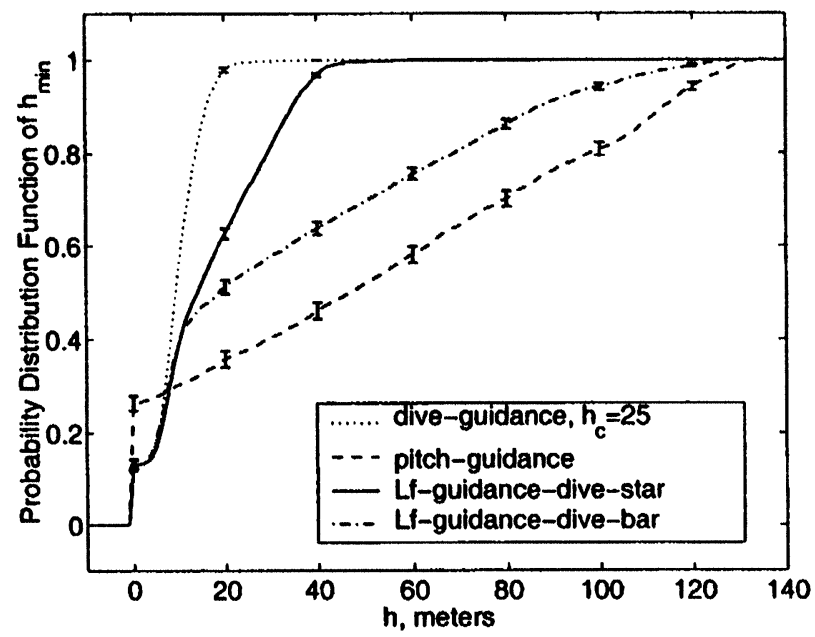

FIGURE 11 Probability distribution function (PDF) of $h_{\min }$, i.e. $\operatorname{Pr}\left(h_{\min } \leq h\right)$ of pitch-, dive- and modified-diveguidance, with $95 \%$ confidence intervals.

$(100-55=45)$; with Lf-guidance-star, 25 times $(80-55=25)$. We say "unnecessarily descended" because descending did not decrease the probability of crash. Thus, Lf-guidancebar has better escape performance than that of both $h$-guidance and Lf-guidance-star.

Now, we compare the PDF of Lf-guidance-bar with that of pitch-guidance at $40 \mathrm{~m}$. The PDF of pitch-guidance at $40 \mathrm{~m}$ is 0.45 . Hence, the aircraft with pitch-guidance has descended lower than $40 \mathrm{~m}$ only 45 times out of 100 microburst encounters. But in these 45 times the aircraft has crashed about 30 times. On the other hand, in 55 times when the aircraft with Lf-guidance-bar has descended lower than $40 \mathrm{~m}$, ground contact or stall has occurred only 15 times. Thus, with pitch-guidance, if the altitude dropped lower than $40 \mathrm{~m}$, the aircraft safely recovered only 15 times $(45-30)$, but with Lf-guidance-bar, 40 times $(55-15)$. 
Similar arguments can be carried out for different altitudes. Hence, Figure 10 clearly shows that using $h^{*}$ or $\bar{h}$ has improved the performance of altitude-guidance, and that the best performance can be obtained with $\bar{h}$ in terms of both probability of crash and probability of minimum altitude being less than a given value.

From Figure 11, the same analysis can be carried out for Lf-guidance-dive-star and Lfguidance-dive-bar. It appears that Lf-guidance-dive-bar, that is, dive-guidance with $\bar{h}$, gives the best performance among the strategies compared in Figure 11. When we compare Lfguidance-bar and Lf-guidance-dive-bar, we can see that Lf-guidance-bar has better performance than that of Lf-guidance-dive-bar. Thus, among all the escape strategies studied within the assumptions of this paper, Lf-guidance-bar appears to be the best when we consider both probability of crash and probability of minimum altitude being less than a given value.

\section{CONCLUSIONS}

We have defined a lift-capability metric, $L_{f}$, that quantifies the aircraft maximum capability of generating an upward force during a microburst encounter. This was done under quasi-static assumptions of longitudinal horizontal flight. In the absence of turbulence, the lift-capability metric has been shown to be a decreasing function of altitude. In the presence of stochastic turbulence, the Lift-capability Factor has been treated as a random variable and its PDF was analytically approximated as a function of altitude. This allowed us to determine the critical altitude that is, the highest altitude where the aircraft can fly with minimum or prespecified probability of danger. Then, altitude-guidance and dive-guidance escape strategies, analyzed in Ref. [14], were modified by using this critical altitude as commanded altitude. Finally, Monte Carlo simulations were conducted to estimate the probability distribution function of minimum altitude reached in a microburst encounter. Using this probability density functions, various escape strategies were comparatively analyzed. In the Monte Carlo simulation, a 6-DOF aircraft model given in Ref. [14] was used.

The deterministic analysis of the Lift-capability Factor has shown that descending to a low altitude in a microburst encounter may improve safety in the sense that the aircraft will have more vertical force capability to maintain its altitude or to climb if necessary. The fact that the lower the recovery altitude the higher the vertical force capability in the absence of turbulence suggests that low altitude flight is more robust in the sense that it is more likely to maintain the altitude despite changes in wind direction and magnitude. Although this conclusion is the same as in Refs. $[5,11]$, the analytical method used in this paper allows us to prove that the lift-capability factor is a decreasing function of altitude and can be used to estimate the critical altitude.

The Probabilistic Analysis Section is used to see whether the conclusion drawn in the Deterministic Analysis Section is valid in the presence of stochastic turbulence. In this section, the Lift-capability Factor is treated as a random variable because it is a function of random variables due to the presence of turbulence. Then, the PDF of the Lift-capability Factor is analytically approximated as a function of altitude. While the Deterministic Analysis Section shows the advantage of descending in a microburst encounter, it completely fails to show that descending too low may increase the risk of failing to have enough vertical force capability to maintain the altitude. However, the probabilistic analysis shows that flying lower increases lift capability up to an optimal altitude, and that flying lower than this optimal value dramatically decreases the lift capability. Thus, the probabilistic analysis has helped analytically corroborate the results of the Monte Carlo simulation in Ref. [14]: the probability 
of crash decreases as the commanded altitude decreases as long as the commanded altitude is higher than an optimal value.

The PDF of the Lift-capability Factor, as a function of altitude, is used to find the critical altitude in a microburst encounter. Critical altitude, in the probabilistic sense, means that the probability of Lift-capability Factor being less than a given value is minimum or less than prespecified value. In other words, using that critical altitude will guarantee that the aircraft flies with minimum or prespecified probability of failing to have enough vertical force capability to maintain altitude. The purpose of flying at the so-called critical altitude is to prevent the aircraft from descending unnecessarily to a fixed altitude in every microburst encounter, but to adjust the recovery altitude depending on the strength of the microburst encountered.

Monte Carlo simulations have shown this purpose can be achieved: specifically, we can decrease the probability of minimum altitude being less than a given value without significantly increasing the probability of crash. That is, in a microburst encounter, a pilot, given the critical altitude, can fly the aircraft at that altitude with minimum or prespecified probability that the aircraft cannot maintain that altitude. This paper provides an analytical method to compute such critical altitudes.

Monte Carlo simulations have also shown that modified altitude guidance, using the critical altitude as the commanded altitude, has better escape performance than both diveguidance with the same procedure and pitch-guidance. Thus, the results of the paper suggests that, during the landing phase of a flight, the critical altitude be monitored, and upon an encounter with a microburst, the aircraft be directed to the last update of the critical altitude and kept there in the high shear region of the microburst.

Besides the conclusion drawn above, the analysis of the PDF of the minimum altitude provides a very simple and decisive criterion to make a comparison between different escape strategies, and shows clearly how to improve the performance of an escape strategy. For example, if the PDF of $h_{\min }$ at an altitude for escape strategy $\mathrm{A}$ is smaller than that for escape strategy B for any altitude, then it can be directly concluded that strategy A has better escape performance than that of strategy B. Moreover, if we are to improve the performance of an escape strategy, the PDF analysis suggests that for a given altitude we should reduce the value of the PDF at that altitude without increasing the PDF at zero altitude. Thus, it appears that the PDF analysis is a very promising tool in microburst studies to develop better escape strategies.

Before practical implementation of this escape procedure, the sensitivity of the procedure with respect to the assumptions should be studied. If the critical altitude is very sensitive to the microburst model, the procedure should be modified with the most accurate model available or additional features should be added to the procedure so that the microburst model can be identified on-board. Similarly, the sensitivity with respect to the turbulence model should be investigated. Also algorithms to estimate wind velocity components and turbulent component of wind should be used. The effect of human factors on the performance of the escape procedure should be studied as well.

\section{References}

[1] Dogan, A. (2000) Guidance Strategies for Microburst Escape, Ph.D. Thesis. Ann Arbor, MI: The University of Michigan.

[2] Windshear Training Aid (1987) Washington, DC: U.S. Department of Transportation, Federal Aviation Administration.

[3] Miele, A., Wang, T., Melvin, W. W. and Bowles, R. L. (1989) Acceleration, gamma, and theta guidance for abort landing in a windshear, J. Guidance, Control, and Dynamics, 12(6), November-December, 815-821.

[4] Visser, H. G. (1997) Minimax optimal control analysis of lateral escape maneuvers for microburst encounters, J. Guidance, Control, and Dynamics, 20(2), March-April, 370-376.

[5] Visser, H. G. (1997) Lateral escape guidance strategies for microburst windshear encounters, J. Aircraft, 34(4), July-August, 514-521. 
[6] Leitmann, G. and Pandey, S. (1990) Aircraft control under conditions of windshear, Proceedings of the 29th Conference on Decision and Control, IEEE, CH2917-3, December, pp. 747-752.

[7] Leitmann, G. and Pandey, S. (1991) Aircraft control for flight in an uncertain environment: Takeoff in windshear, J. Optimization Theory and Applications, 70(1), July, 25-55.

[8] Leitmann, G. and Pandey, S. (1991) Adaptive control of aircraft in windshear, Proceedings of the 30th Conference on Decision and Control, IEEE CH3076-7, December, pp. 2157-2161.

[9] Stalford, H. (1991) Mu control of aircraft in windshear, The IEEE Aerospace \& Electronic System Society, 1991 National Telesystems Conference, IEEE, CH3010-6, March, pp. 19-24.

[10] Prasanth, R. K., Bailey J. E. and Krishnakumar, K. (1992) Robust wind shear stochastic controller-estimator, J. Guidance, Control, and Dynamics, May-June.

[11] Zhao, Y. and Bryson, A. E., Jr. (1990) Optimal paths through downbursts, J. Guidance, Control, and Dynamics, 13(5), 813-818.

[12] Avila de Melo, D. and Hansman, R. J. (1991) Analysis of aircraft performance during lateral maneuvering for microburst avoidance, J. Aircraft., December.

[13] Dogan, A. and Kabamba, P. T. (1998) Microburst escape using altitude guidance, Proceedings of the 37th IEEE Conference on Decision \& Control, Tampa, FL, December, pp. 4228-4233.

[14] Dogan, A. and Kabamba, P. T. (2000) Escaping a microburst with turbulence: Altitude, dive and pitch guidance strategies, J. Aircraft, 37(3), 417-426.

[15] Roskam, Jan (1995) Airplane Flight Dynamics and Automatic Flight Controls. DAR Corporation.

[16] Boeing 747-400 Airplane Flight Manual, D6U10001, Boeing Co., January, 1989.

[17] Donald McLean (1990) Automatic Flight Control Systems. Prentice Hall International (UK) Ltd.

[18] Campbell, C. W. (1984) A spatial model of wind shear and turbulence for flight simulation, NASA TP-2313.

[19] Friedland, B. (1984) Control System Design. New York: McGraw-Hill, Inc.

[20] Stark, H. and Woods, J. W. (1994) Probability, Random processes, and Estimation Theory for Engineers. Upper Saddle River, NJ: Prentice-Hall, Inc.

\section{A APPENDIX}

The following is a well-known property of linear-stochastic systems [19]. Consider the following linear time-invariant system

$$
\begin{aligned}
& \dot{x}=A x+B \eta, \\
& y=C x,
\end{aligned}
$$

where $\eta$ is a Gaussian white-noise process vector such that

$$
\begin{aligned}
E[\eta(t)] & =0, \\
E\left[\eta(t) \eta^{\mathrm{T}}(\tau)\right] & =R_{\eta} \delta(t-\tau),
\end{aligned}
$$

where $R_{\eta}$, is a constant matrix. If the linear system is asymptotically stable, then at steadystate the state, $x$, is a Gaussian random process with

$$
\begin{aligned}
E[x(t)] & =0, \\
E\left[x(t) x^{\mathrm{T}}(t)\right] & =R_{x},
\end{aligned}
$$

where the covariance matrix of $x, R_{x}$ satisfies the following Lypunov equation

$$
A R_{x}+R_{x} A^{\mathrm{T}}+B R_{\eta} B^{\mathrm{T}}=0 .
$$

Moreover, at steady-state

$$
\begin{aligned}
E[y(t)] & =0, \\
E\left[y(t) y^{\mathrm{T}}(t)\right] & =C R_{x} C^{\mathrm{T}} .
\end{aligned}
$$


From this property, it follows that $u_{g}(t)$, whose dynamics is given in Eq. (24), is a Gaussian random process with zero mean and variance

$$
\operatorname{var}\left(u_{g}\right)=\frac{\sigma^{2} K_{u}}{2 \lambda_{u}}
$$

where $K_{u}=2 V \sigma_{u}^{2} /\left(L_{u} \pi\right)$ and $\lambda_{u}=V / L_{u}$. Thus

$$
\operatorname{var}\left(u_{g}\right)=\frac{\sigma^{2}}{\pi} \sigma_{u}^{2}
$$

The assumption of isotropic turbulence implies [17] that

$$
\frac{\sigma_{u}^{2}}{L_{u}}=\frac{\sigma_{w}^{2}}{L_{w}}
$$

This equation along with Eqs. (69) and (26) yields Eq. (45). Similarly for $w_{g}$, whose dynamics is given in Eq. (25),

$$
\operatorname{var}\left(w_{g}\right)=\frac{K_{w} \sigma^{2}}{4 \lambda_{w}}\left(\frac{\beta_{w}^{2}}{\lambda_{w}^{2}}+1\right),
$$

where $K_{w}=3 \sigma_{w}^{2} V /\left(L_{w} \pi\right), \lambda_{w}=V / L_{w}$, and $\beta_{w}=V /\left(\sqrt{3} L_{w}\right)$. Substituting these parameters into Eq. (71) yields Eq. (46).

\section{B APPENDIX}

From Eqs. (52), (42), (39), (21), (22), and (23), we have

$$
\begin{gathered}
L_{f}=L_{f}\left(V, \delta_{e}^{*}, W_{x}, W_{h}\right), \\
V=V\left(W_{x}, W_{h}\right), \\
\delta_{e}^{*}=\delta_{e}^{*}(V), \\
W_{x}=W_{x}\left(d_{x}\right), \\
W_{h}=W_{h}\left(d_{h}\right), \\
d_{x}=d_{x}\left(u_{g}, w_{g}\right), \\
d_{h}=d_{h}\left(u_{g}, w_{g}\right),
\end{gathered}
$$

where only functional dependence on random variables is shown.

Using the above functional dependence, the partial derivatives are taken and evaluated at $\left(u_{g}, w_{g}\right)=(0,0)$. Thus, we obtain

$$
\begin{aligned}
\left.\frac{\partial L_{f}}{\partial u_{g}}\right|_{(0,0)}= & \left.\left.\frac{\partial L_{f}}{\partial V}\right|_{(0,0)} \frac{\partial V}{\partial u_{g}}\right|_{(0,0)}+\left.\left.\frac{\partial L_{f}}{\partial \delta_{e}^{*}}\right|_{(0,0)} \frac{\partial \delta_{e}^{*}}{\partial u_{g}}\right|_{(0,0)} \\
& +\left.\left.\frac{\partial L_{f}}{\partial W_{x}}\right|_{(0,0)} \frac{\partial W_{x}}{\partial u_{g}}\right|_{(0,0)}+\left.\left.\frac{\partial L_{f}}{\partial W_{h}}\right|_{(0,0)} \frac{\partial W_{h}}{\partial u_{g}}\right|_{(0,0)},
\end{aligned}
$$




$$
\begin{aligned}
\left.\frac{\partial L_{f}}{\partial w_{g}}\right|_{(0,0)}= & \left.\left.\frac{\partial L_{f}}{\partial V}\right|_{(0,0)} \frac{\partial V}{\partial w_{g}}\right|_{(0,0)}+\left.\left.\frac{\partial L_{f}}{\partial \delta_{e}^{*}}\right|_{(0,0)} \frac{\partial \delta_{e}^{*}}{\partial w_{g}}\right|_{(0,0)} \\
& +\left.\left.\frac{\partial L_{f}}{\partial W_{x}}\right|_{(0,0)} \frac{\partial W_{x}}{\partial w_{g}}\right|_{(0,0)}+\left.\left.\frac{\partial L_{f}}{\partial W_{h}}\right|_{(0,0)} \frac{\partial W_{h}}{\partial w_{g}}\right|_{(0,0)},
\end{aligned}
$$

$$
\begin{aligned}
\left.\frac{\partial L_{f}}{\partial V}\right|_{(0,0)}= & \frac{1}{2 W} \rho \mathcal{S}\left[\left\{C_{L_{0}}+C_{L_{\alpha}} \alpha_{\max }+C_{L_{\delta_{e}}} \delta_{e}^{*}\left(V^{d}\right)\right\}\left\{V_{A / C}-W_{x}^{d}\right\}\right. \\
& \left.-\left\{C_{D_{0}}+C_{D_{\alpha}} \alpha_{\max }\right\} W_{h}^{d}\right]+\frac{T_{1}+2 T_{2} V^{d}}{W} \sin \theta^{*}, \\
& \left.\frac{\partial L_{f}}{\partial \delta_{e}^{*}}\right|_{(0,0)}=\frac{1}{2 W} \rho \mathcal{S} C_{L_{\delta_{e}}} V^{d}\left(V_{A / C}-W_{x}^{d}\right),
\end{aligned}
$$$$
\left.\frac{\partial L_{f}}{\partial W_{x}}\right|_{(0,0)}=-\frac{1}{2 W} \rho V^{d} \mathcal{S}\left\{C_{L_{0}}+C_{L_{\alpha}} \alpha_{\max }+C_{L_{\delta_{e}}} \delta_{e}^{*}\left(V^{d}\right)\right\}
$$$$
\left.\frac{\partial L_{f}}{\partial W_{h}}\right|_{(0,0)}=-\frac{1}{2 W} \rho V^{d} \mathcal{S}\left\{C_{D_{0}}+C_{D_{\alpha}} \alpha_{\max }\right\}
$$$$
\left.\frac{\partial V}{\partial u_{g}}\right|_{(0,0)}=-\frac{\left(V_{A / C}-W_{x}^{d}\right)}{V^{d}} \cos \theta^{*}+\frac{W_{h}^{d}}{V^{d}} \sin \theta^{*}
$$$$
\left.\frac{\partial \delta_{e}^{*}}{\partial u_{g}}\right|_{(0,0)}=\left.\left.\frac{\partial \delta_{e}^{*}}{\partial V}\right|_{(0,0)} \frac{\partial V}{\partial u_{g}}\right|_{(0,0)},
$$$$
\left.\frac{\partial \delta_{e}^{*}}{\partial V}\right|_{(0,0)}=\frac{2 \Delta z}{C_{\mathcal{M}_{\delta_{e}}} \rho \mathcal{S}_{\bar{c}}} \frac{1}{V^{d^{2}}}\left(\frac{2 T_{0}}{V^{d}}+T_{1}\right)
$$$$
\left.\frac{\partial W_{x}}{\partial u_{g}}\right|_{(0,0)}=\cos \theta^{*}
$$$$
\left.\frac{\partial W_{h}}{\partial u_{g}}\right|_{(0,0)}=\sin \theta^{*}
$$

$$
\begin{gathered}
\left.\frac{\partial V}{\partial w_{g}}\right|_{(0,0)}=-\frac{\left(V_{A / C}-W_{x}^{d}\right)}{V^{d}} \sin \theta^{*}-\frac{W_{h}^{d}}{V^{d}} \cos \theta^{*} \\
\left.\frac{\partial \delta_{e}^{*}}{\partial w_{g}}\right|_{(0,0)}=\left.\left.\frac{\partial \delta_{e}^{*}}{\partial V}\right|_{(0,0)} \frac{\partial V}{\partial w_{g}}\right|_{(0,0)} \\
\left.\frac{\partial W_{x}}{\partial w_{g}}\right|_{(0,0)}=\sin \theta^{*} \\
\left.\frac{\partial W_{h}}{\partial w_{g}}\right|_{(0,0)}=-\cos \theta^{*}
\end{gathered}
$$




\section{APPENDIX}

Let $X(h)$ be a Gaussian random variable with mean $\mu_{X}(h)$ and variance $\sigma_{X}^{2}(h)$. The probability of $X(h)$ being less than or equal to $x$ is

$$
\operatorname{Pr}[X \leq x]=\frac{1}{2}\left[1+\operatorname{erf}\left(\frac{x-\mu_{X}}{\sqrt{2 \sigma_{X}^{2}}}\right)\right]
$$

(i) Finding $h^{*}$

By definition, $h^{*}$ is

$$
h^{*}=\arg \min _{h} \operatorname{Pr}[X \leq x]
$$

To find $h^{*}$ from Eq. (94), we need to solve the following equation for $h$

$$
\frac{\partial \operatorname{Pr}[X \leq x]}{\partial h}=0
$$

Substituting Eq. (94) into Eq. (96) and taking the partial derivative with respect to $h$, we have

$$
\frac{\partial}{\partial h}\left\{\operatorname{erf}\left(\frac{x-\mu_{X}}{\sqrt{2 \sigma_{X}^{2}}}\right)\right\}=0 .
$$

Since $\mu_{X}$ and $\sigma_{X}^{2}$ are functions of $h$, the above equation is equivalent to

$$
\frac{\partial}{\partial \mu_{X}}\left\{\operatorname{erf}\left(\frac{x-\mu_{X}}{\sqrt{2 \sigma_{X}^{2}}}\right)\right\} \frac{\partial \mu_{X}}{\partial h}+\frac{\partial}{\partial \sigma_{X}^{2}}\left\{\operatorname{erf}\left(\frac{x-\mu_{X}}{\sqrt{2 \sigma_{X}^{2}}}\right)\right\} \frac{\partial \sigma_{X}^{2}}{\partial h}=0,
$$

where, recall that

$$
\operatorname{erf}\left(\frac{x-\mu_{X}}{\sqrt{2 \sigma_{X}^{2}}}\right)=\frac{2}{\sqrt{\pi}} \int_{0}^{\left(x-\mu_{X}\right) / \sqrt{2 \sigma_{X}^{2}}} \exp \left(-\gamma^{2}\right) \mathrm{d} \gamma
$$

By Leibnitz's Rule, the partial derivatives in Eq. (98) are

$$
\begin{aligned}
& \frac{\partial}{\partial \mu_{X}}\left\{\operatorname{erf}\left(\frac{x-\mu_{X}}{\sqrt{2 \sigma_{X}^{2}}}\right)\right\}=-\frac{1}{\sqrt{2 \sigma_{X}^{2}}} \exp \left(-\frac{\left(x-\mu_{X}\right)^{2}}{2 \sigma_{X}^{2}}\right), \\
& \frac{\partial}{\partial \sigma_{X}^{2}}\left\{\operatorname{erf}\left(\frac{x-\mu_{X}}{\sqrt{2 \sigma_{X}^{2}}}\right)\right\}=-\frac{x-\mu_{X}}{\left(2 \sigma_{X}^{2}\right)^{3 / 2}} \exp \left(-\frac{\left(x-\mu_{X}\right)^{2}}{2 \sigma_{X}^{2}}\right) .
\end{aligned}
$$

Substituting (100) and (101) into (98) and rearranging, we obtain

$$
-\frac{1}{\left(2 \sigma_{X}^{2}\right)^{1 / 2}} \exp \left(-\frac{\left(x-\mu_{X}\right)^{2}}{2 \sigma_{X}^{2}}\right)\left\{\frac{\partial \mu_{X}}{\partial h}+\frac{\left(x-\mu_{X}\right)}{2 \sigma_{X}^{2}} \frac{\partial \sigma_{X}^{2}}{\partial h}\right\}=0
$$


where $\mu_{X}$ and $\sigma_{X}^{2}$ are assumed to be positive finite. Thus, the above equation is equivalent to

$$
\frac{\partial \mu_{X}(h)}{\partial h}+\frac{\left(x-\mu_{X}(h)\right)}{2 \sigma_{X}^{2}(h)} \frac{\partial \sigma_{X}^{2}(h)}{\partial h}=0 .
$$

The altitude $h$ that solves this equation is $h^{*}$. Although it is not easy to obtain explicitly an analytic expression for $h^{*}$, it can be solved numerically.

(ii) Finding $\bar{h}$

By definition, $\bar{h}$ is, for a given $\operatorname{Pr}$, such that

$$
\operatorname{Pr}[X(\bar{h}) \leq x]=\operatorname{Pr}
$$

From Eq. (94), we have

$$
\frac{1}{2}\left[1+\operatorname{erf}\left(\frac{x-\mu_{X}(\bar{h})}{\sqrt{2 \sigma_{X}^{2}(\bar{h})}}\right)\right]=\operatorname{Pr}
$$

which implies

$$
\operatorname{erf}\left(\frac{x-\mu_{X}(\bar{h})}{\sqrt{2 \sigma_{X}^{2}(\bar{h})}}\right)=2 \operatorname{Pr}-1
$$

which implies

$$
\frac{x-\mu_{X}(\bar{h})}{\sqrt{2 \sigma_{X}^{2}(\bar{h})}}=\operatorname{erf}^{-1}(2 \operatorname{Pr}-1),
$$

where $\operatorname{erf}^{-1}(\cdot)$ is the inverse error function. This equation also can be solved numerically for $\bar{h}$. 


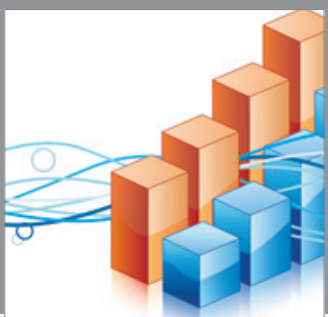

Advances in

Operations Research

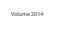

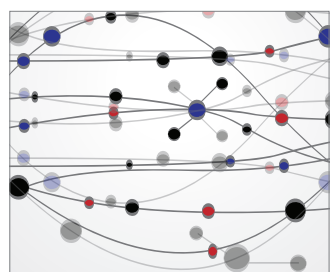

\section{The Scientific} World Journal
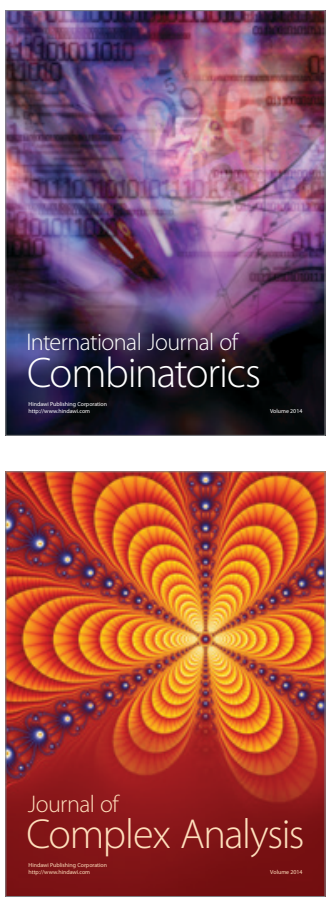

International Journal of

Mathematics and

Mathematical

Sciences
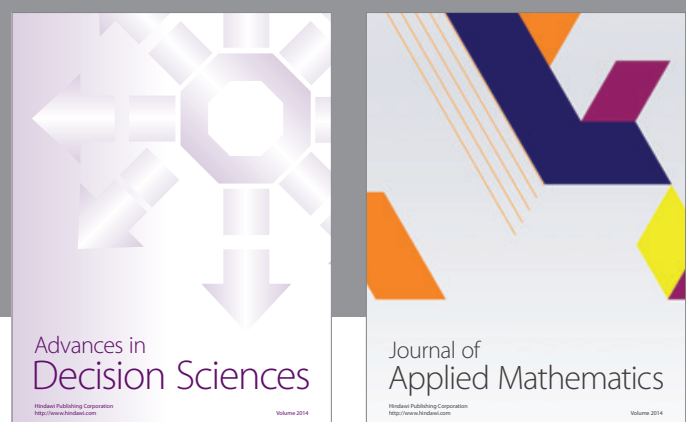

Journal of

Applied Mathematics
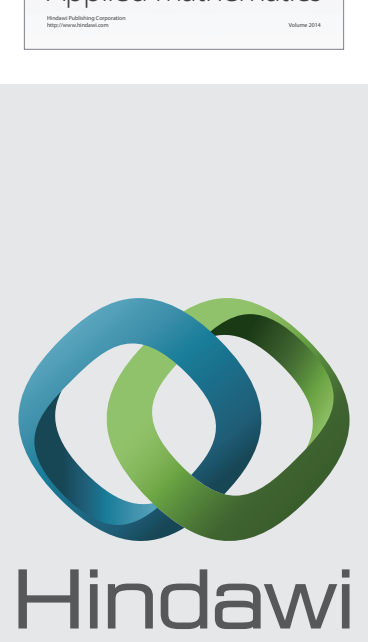

Submit your manuscripts at http://www.hindawi.com
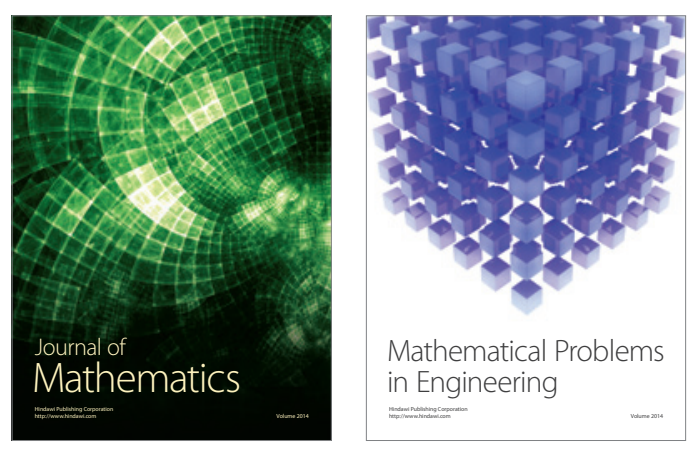

Mathematical Problems in Engineering
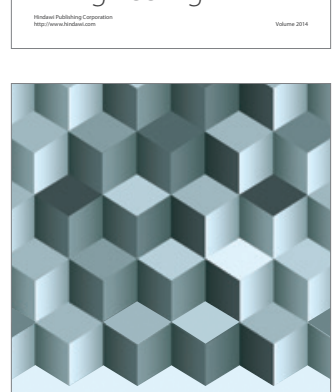

Journal of

Function Spaces
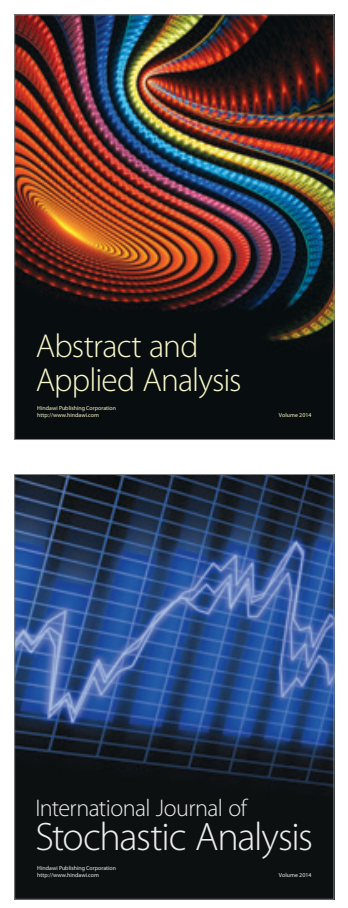

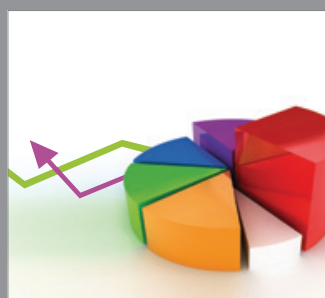

ournal of

Probability and Statistics

Promensencen
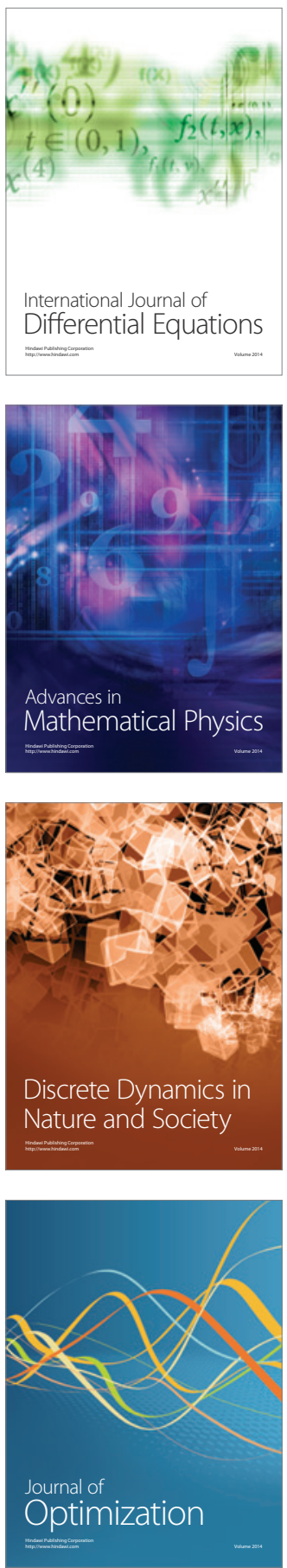